\title{
Indicators of mineralocorticoid excess in the evaluation of primary aldosteronism
}

\author{
Melania Balaş ${ }^{1,2}$, Ioana Zosin ${ }^{2}$, Christiane Maser-Gluth ${ }^{3}$, Derik Hermsen ${ }^{4}$, Kenko Cupisti ${ }^{5}$, Matthias Schott ${ }^{1}$, \\ Sven Schinner ${ }^{1}$, Wolfram T Knoefel ${ }^{5}$, Werner A Scherbaum ${ }^{1}$ and Holger S Willenberg ${ }^{1}$
}

It is suggested to use the aldosterone-to-renin ratio (ARR) as a first test in the screening for primary aldosteronism (PA). However, many groups rather rely on the determination of urinary tetrahydroaldosterone secretion; others calculate a ratio of urinary aldosterone to plasma renin activity. The aim of the present study was to evaluate the usefulness of different parameters of aldosterone excess in the case finding of PA. The study included 28 patients with PA and 33 subjects with essential hypertension. Clinical data, which included the hormonal parameters, serum aldosterone, plasma renin concentration, urinary free aldosterone and metabolites and serum and urinary electrolyte levels were analyzed. These indices of aldosterone excess, the ARR, serum sodium to urinary sodium to (serum potassium) ${ }^{2}$ to urinary potassium (SUSPPUP) ratio and combinations of these parameters were compared between the groups. Receiver-operating curve analysis revealed that the ARR multiplied by the SUSPPUP ratio (ARR $\times$ SUSPPUP) is the most reliable screening test, with a sensitivity of $92.3 \%$ and a specificity of $93.9 \%$ (cutoff point $\left.199.2\left(\mathrm{mmol} \mathrm{I}^{-1}\right)^{-1}\right)$. The combination of ARR $\times$ SUSPPUP ratio with urinary free aldosterone divided by the plasma renin concentration rendered a specificity of $100 \%$. Less useful was the correction of urinary free aldosterone and its metabolites for sodium excretion. Although the ARR and urinary free aldosterone divided by renin are good tests in the screening for PA, the combination of ARR with SUSPPUP ratio is a better indicator of an aldosterone excess and aldosterone action in patients with ongoing antihypertensive medication. Antihypertensive drugs only marginally interfere with the SUSPPUP ratio, but they may influence the ARR, whereby the effects in PA patients seem to be negligible.

Hypertension Research (2010) 33, 850-856; doi:10.1038/hr.2010.76; published online 3 June 2010

Keywords: adrenal; aldosterone; Conn syndrome; primary aldosteronism; SUSPPUP

\section{INTRODUCTION}

The detection of primary aldosteronism (PA) among hypertensive patients is a prominent task, because PA is now considered to be the most frequent form of secondary hypertension and is potentially reversible with specific therapeutical approaches. ${ }^{1}$ Using the aldosteroneto-renin ratio (ARR) as a screening tool, several studies found that PA is more frequent than previously assumed, affecting $1-13 \%$ of hypertensive individuals. ${ }^{2-6}$ The different frequency found in these studies is because of the use of different screening tools, study designs and various study populations.

A considerable portion of PA patients have normal serum potassium levels. ${ }^{7}$ Therefore, in the diagnosis of PA, hypokalemia has low sensitivity and specificity and is not anymore suggested as a criterion to make the diagnosis of PA.,

The plasma aldosterone-to-plasma renin activity $\left(\mathrm{A}_{\mathrm{PL}} / \mathrm{PRA}\right)$ ratio has proved to be one of the most reliable parameters in the screening for PA. Many studies have shown superior sensitivity and specificity of the $A_{P L} / P R A$ ratio, as compared with measurements of $A_{P L}$ or renin alone. However, the $A_{P L} / P R A$ ratio is influenced by numerous factors and sampling conditions, including age, sodium intake, time of day when the samples are collected, posture, serum potassium concentration, kidney function and medication. ${ }^{9-12}$ Other studies indicated that $A_{P L} / P R A$ is a valid screening test also in patients with ongoing antihypertensive treatment. ${ }^{13}$ As many factors affect the $A_{P L} / P R A$, it was recommended as a screening test only and it should be repeated if initial results are inconclusive. ${ }^{1}$

Overall, the predictive value of the ARR is hampered, as aldosterone is also regulated by factors other than angiotensin II and normal values of $A_{P L}$ can be amplified by very low PRA levels, leading to a high $A_{P L} /$ PRA ratio. ${ }^{14}$ As stated by Young and others, if $A_{P L}$ is included as a second screening test the accuracy increases. ${ }^{15,16}$ The logistic regression model developed, based on $\mathrm{A}_{\mathrm{PL}}$ and PRA, showed a

\footnotetext{
${ }^{1}$ Department of Endocrinology, Diabetes and Rheumatology, University Hospital Duesseldorf, Duesseldorf, Germany; ${ }^{2}$ Department of Endocrinology, University of Medicine and Pharmacy 'Victor Babeş', Timişoara, Romania; ${ }^{3}$ Department of Pharmacology, University of Heidelberg, Heidelberg; Germany; ${ }^{4}$ Central Institute for Clinical Chemistry and Laboratory Medicine, University Hospital Duesseldorf, Duesseldorf, Germany and ${ }^{5}$ Department of General, Visceral, and Pediatric Surgery, University Hospital Duesseldorf, Duesseldorf, Germany

Correspondence: Dr HS Willenberg, Department of Endocrinology, Diabetes and Rheumatology, University Hospital Duesseldorf, Moorenstrasse 5, D-40225 Duesseldorf, Germany. E-mail: Holger.Willenberg@uni-duesseldorf.de
}

Received 5 October 2009; revised 15 March 2010; accepted 5 April 2010; published online 3 June 2010 
good sensitivity and specificity. To simplify the model and to facilitate the use in clinical practice, $A_{P L} \times A R R\left(A^{2} R R\right)$ and $A_{P L}{ }^{2} \times A R R\left(A^{3} R R\right)$ were proposed with improved accuracy. ${ }^{17}$

Aldosterone is excreted in the urine in three main forms: aldosterone-18-glucuronide (A18G), urinary unconjugated (free) aldosterone $\left(A_{U R}\right)$ and tetrahydroaldosterone (THA), which reflects up to $45 \%$ of aldosterone secretion. ${ }^{18}$ Urine analysis $(24 \mathrm{~h})$ for aldosterone metabolites eliminates most of the posture fluctuations in aldosterone blood values, diurnal variations and episodic secretion of aldosterone by the adrenal glands. The urinary unconjugated (free) aldosterone-to-active renin ratio $\left(A_{U R} / R\right)$ proved to be a good independent predictor for surgical curability after adrenalectomy, in subjects with aldosteroneproducing adenomas (APAs). ${ }^{19,20}$

Recently, our group has shown the usefulness of the serum sodium to urinary sodium to (serum potassium) ${ }^{2}$ to urinary potassium (SUSPPUP) ratio in the diagnosis of PA. When calculated from synchronous measurements of sodium and potassium in serum as well as in urine in a fasting state, the SUSPPUP ratio reflects the mineralocorticoid excess and was proposed as a cheap and rapid tool in the screening for PA. ${ }^{21}$

The present study was performed to assess the usefulness of different parameters in the first line screening for PA, including correction for plasma renin concentration or/and urinary sodium excretion.

\section{METHODS}

\section{Subjects}

The study group comprised 28 patients with PA and 33 patients with essential hypertension (EH). The majority of patients were under therapy with antihypertensive drugs when blood and urinary samples were collected. Patients with PA took $\beta$-blockers (BBL) or clonidine (Clon) in $61 \%$ of cases, angiotensin-converting enzyme inhibitors (ACEIs) or angiotensin receptor blockers (ARBs) in 57\%, hydrochlorothiazide (HCT) in 32\% and/or amlodipine in $75 \%$. Patients with EH had slightly better blood pressure values (Table 1) despite taking less antihypertensive agents (BBL or Clon in $27 \%$ of cases, ACEIs or ARBs in 26\%, HCT in 18\% and amlodipine in 26\%). However, all drugs known to interfere with the mineralocorticoid receptor or the epithelial sodium channel, as well as loop diuretics, were discontinued for 6 weeks before inclusion of patients into our study. There was no restriction on dietary sodium intake; patients were rather asked to follow a sodium-enriched diet during the diagnostic procedures. Potassium supplementation aimed at keeping serum potassium concentrations around $4 \mathrm{mmoll}^{-1}$ and was introduced only after the SUSPPUP and urine analyses or paused for 3 days before sampling.

Table 1 Characteristics of the studied groups, values expressed as mean $\pm \mathbf{s}$.d. and significance of differences between the groups, with statistical tests specified below

\begin{tabular}{|c|c|c|c|}
\hline Parameter & $P A($ mean \pm s.d.) & $E H($ mean \pm s.d. $)$ & P-value \\
\hline Number $(n)$ & 28 & 33 & \\
\hline Females/males $(n)$ & $16 / 12$ & $24 / 9$ & $0.28^{a}$ \\
\hline Age (year) & $55.71 \pm 9.11$ & $52.41 \pm 11.29$ & $0.32^{b}$ \\
\hline $\mathrm{SBP}(\mathrm{mm} \mathrm{Hg})$ & $154.8 \pm 23.4$ & $148.9 \pm 23.31$ & $0.35^{c}$ \\
\hline DBP (mm Hg) & $92 \pm 13.2$ & $90.33 \pm 12.7$ & $0.63^{c}$ \\
\hline Serum potassium $\left(\mathrm{mmol} \mathrm{I}^{-1}\right)$ & $3.41 \pm 0.72$ & $4,07 \pm 0.66$ & $<0.0001^{\mathrm{c}}$ \\
\hline Urinary sodium (mmol per $24 \mathrm{~h}$ ) & $171.0 \pm 76.1$ & $167.8 \pm 91.8$ & $0.88^{b}$ \\
\hline
\end{tabular}

Abbreviations: DBP, diastolic blood pressure; EH, essential hypertension; PA, primary aldosteronism. SBP, systolic blood pressure.

aldosteronism; SBP, sy

bUnpaired Student's $t$-test.

'Nonparametric Mann-Whitney test.
Among the PA patients, 15 (53.6\%) had an APA, confirmed by computed tomography and adrenal venous sampling; the others had bilateral adrenal disease and were classified as having idiopathic hyperaldosteronism.

\section{Methods}

The patients were included in the PA group based on the following criteria: (a) low serum renin concentration associated with a relative high serum aldosterone level, an elevated ARR on one occasion (for example, before or at admittance) and (b) one positive confirmatory test (intravenous saline infusion or fludrocortisone suppression test), following the recommendations as described in the Endocrine Society Clinical Practice Guideline. ${ }^{1}$ Thus, PA was diagnosed in patients with a high ARR of $>38\left[\mathrm{ngl}^{-1}: \mathrm{ngl}^{-1}\right]$ and an inadequate high $\mathrm{A}_{\mathrm{PL}}$ of $>60 \mathrm{ng} \mathrm{l}^{-1}$ after fludrocortisone testing or $>100 \mathrm{ng} \mathrm{l}^{-1}$ after the saline infusion test. ${ }^{1}$ The diagnosis of $\mathrm{EH}$ was established if other causes of hypertension were excluded. With the subjects seated for at least $15 \mathrm{~min}$ in the morning, the blood pressure was measured and blood samples were taken.

Serum aldosterone was determined by Coat-A-Count radioimmunoassay (DPC Siemens, Bad Nauheim, Germany). To obtain aldosterone in SI units $\left(\mathrm{nmoll}^{-1}\right)$, values in $\mathrm{ng} \mathrm{l}^{-1}$ should be multiplied by 0.0277 . Serum active renin concentration was determined by radioimmunoassay (DiaSorin, Saluggia, Italy). For conversion of renin in $\mathrm{pmoll}^{-1}$, renin concentration in $\mathrm{ngl}^{-1}$ should be multiplied by 0.0237 . The daily sodium intake was estimated by 24 -h urinary sodium excretion and expressed as mmol per $24 \mathrm{~h}$. The SUSPPUP ratio was calculated from synchronous measurements of sodium and potassium in serum and urine in a fasting state based on the formula, serum sodium/ urinary sodium divided by (serum potassium) $)^{2}$ urinary potassium..$^{21}$ The ratio is expressed in $\left(\mathrm{mmoll}^{-1}\right)^{-1}$. The urinary sodium and potassium concentrations were determined from the sample of the 24-h urine. Electrolytes have been measured in serum and urine using ISE (Roche, Mannheim, Germany).

All the other urinary hormonal parameters (THA, $A_{U R}$ and A18G) were determined by radioimmunoassay after chromatographic purification, in a 24-h urine sample and expressed as $\mu \mathrm{g}$ per $24 \mathrm{~h} .^{22,23}$

The ARR was calculated as follows: serum aldosterone concentration $\left(\mathrm{ng}^{-1}\right)$ divided by the plasma active renin concentration $\left(\mathrm{ngl}^{-1}\right)$. The correction for sodium intake or renin was performed by multiplying the urinary free aldosterone and metabolites concentrations by $24 \mathrm{~h}$ urinary sodium excretion or the plasma renin concentration, respectively.

To analyze the effect of different antihypertensive agents, data of patients with PA $(n=52)$ or EH $(n=89)$ was collected and several groups formed, whereby patients with PA were separated from those with EH, and patients taking one particular antihypertensive drug were compared with those who did not.

\section{Statistical analysis}

Statistical values are reported as mean \pm standard deviation (s.d.) (or standard error of the mean (s.e.m.) when indicated). Normal distribution of the parameters was evaluated using the Kolmogorov-Smirnov test. Two-tailed Student's $t$-test or nonparametric Mann-Whitney test were used for comparison of continuous variables between the groups, as appropriate. Specificity and sensitivity were calculated using receiver-operating characteristic curves. Correlation between continuous variables was assessed using Pearson's correlation coefficient or Spearman's rank correlation coefficient, as indicated. The analysis of categorical data was carried out using contingency table and Fisher's exact test. The positive predictive value of the test was defined as the number of true positive subjects divided by the number of all positive subjects for the test. The negative predictive value was defined as the ratio between the number of true negative subjects and the number of all negative subjects for the test.

A $P$-value below 0.05 was considered statistically significant. Statistical analysis was performed using GraphPad Prism for Windows, versions 4 and 5, statistical software (GraphPad Software, San Diego, CA, USA).

\section{RESULTS}

The baseline characteristics of the studied patients are shown in Table 1. There were no statistical differences regarding age, systolic blood pressure or diastolic blood pressure between the groups. 
Table 2 Main results of the laboratory studies

\begin{tabular}{|c|c|c|c|}
\hline Parameter & $P A(m e a n \pm$ s.d.) & $E H(m e a n \pm s . d)$. & P-value \\
\hline$A_{P L}\left(n g I^{-1}\right) ;$ median & $282.0 \pm 170.1 ; 270.0$ & $101.0 \pm 97.9 ; 65.0$ & $<0.001$ \\
\hline$A_{U R}(\mu g$ per $24 \mathrm{~h}$ ); median & $0.85 \pm 0.68 ; 0.57$ & $0.24 \pm 0.16 ; 0.22$ & $<0.001$ \\
\hline THA ( $\mu$ g per $24 \mathrm{~h}$ ); median & $173.0 \pm 119.8 ; 150.0$ & $57.7 \pm 34.6 ; 50.0$ & $<0.001$ \\
\hline A18G ( $\mu$ g per $24 \mathrm{~h}$ ); median & $22.1 \pm 22.7 ; 18.5$ & $8.37 \pm 8.7 ; 5.0$ & 0.001 \\
\hline ARR $\times \operatorname{SUSPPUP}\left(\left(\mathrm{mmol} \mathrm{I}^{-1}\right)^{-1}\right) ;$ median & $1196.8 \pm 1177.7 ; 741.0$ & $63.7 \pm 77.1 ; 34.6$ & $<0.0001$ \\
\hline
\end{tabular}

Abbreviations: A18G, aldosterone-18-glucuronide; $A_{P L}$, plasma aldosterone; ARR aldosterone to renin ratio; $A_{U R}$, urinary unconjugated (free) aldosterone; $A_{U R} / R$, urinary unconjugated (free) aldosterone to renin ratio; EH, essential hypertension; PA, primary aldosteronism; R, plasma renin concentration; SUSPPUP, serum sodium to urinary sodium divided by the (serum potassium) ${ }^{2}$ to urinary potassium ratio; THA, tetrahydroaldosterone.

The Student's $t$-test was used for comparison of the THA and AUR/R data between the groups; for other parameters, comparison between the groups was carried out using the nonparametric Mann-Whitney test. The significance of differences between the studied groups, regarding laboratory parameters, was expressed as mean \pm s.d. and median values.

Hypokalemia was present in $75 \%$ of $\mathrm{PA}$ patients and in $27 \%$ of $\mathrm{EH}$ patients $(P<0.0001$; Fisher's exact test), and serum potassium concentrations differed significantly between the two groups (Table 1). There were no statistically significant differences with regard to 24-h urinary sodium excretion ( $P=0.88$; unpaired $t$-test). Among the $\mathrm{EH}$ patients, three presented with renin values below the lower limit of detection. The main results of the laboratory studies are given in Table 2.

A positive and significant correlation between THA and $\mathrm{A}_{\mathrm{PL}}$ values was noted in all study patients (Spearman's correlation coefficient $r=0.564 ; P<0.0001)$, with a stronger correlation in the PA group (Pearson's correlation coefficient $r=0.634, r^{2}=0.402 ; P<0.0001$ ).

Diastolic blood pressure values correlated positively with $A_{U R}$ in PA patients (Spearman's correlation coefficient $r=0.515 ; P=0.02$ ). Systolic blood pressure values correlated weakly with $A_{U R}$ values in PA patients (Spearman's correlation coefficient $r=0.320 ; P=0.19$ ). In the $\mathrm{EH}$ group, blood pressure values did not correlate with $A_{U R}$ concentration. In both groups, there were no statistically significant correlations found between blood pressure values and other hormonal parameters.

All the investigated parameters, including the 'optimal' cutoff values, area under the receiver-operating curves (AUC), 95\% confidence intervals ( $95 \% \mathrm{CI}$ ), sensitivities, specificities, positive predictive values and negative predictive values, are given in Table 3.

At a cutoff point of $199.2\left(\mathrm{mmoll}^{-1}\right)^{-1}, \mathrm{ARR} \times$ SUSPPUP revealed a good accuracy $(90.1 \%)$, with a high positive predictive value $(92.3 \%)$. If the cutoff value was $62.1\left(\mathrm{mmoll}^{-1}\right)^{-1}$, all the PA patients would have been identified (100\% sensitivity), but $10 \mathrm{EH}$ patients assumed to have PA (69.7\% specificity). The distribution within the patients groups and a receiver-operating curve plot for ARR $\times$ SUSPPUP ratio is presented in Figure 1 . The ARR $\times$ SUPPUP values correlated well with the urinary aldosterone excretion rates and were best with the combination of THA and A18G (Table 4).

In hypokalemic patients, ARR $\times$ SUSPPUP showed a sensitivity of $94.7 \%$ (95\% CI $73.9-99.8 \%$ ) and specificity of $88.8 \%$ (95\% CI $51.7-99.7 \%$ ) at a cutoff value of $199.2\left(\mathrm{mmoll}^{-1}\right)^{-1}$ (AUC is 0.976 , s.e.m. 0.02 and $95 \%$ CI $0.93-1.02$ ). In normokalemic patients, the ARR $\times$ SUSPPUP values displayed a slightly higher specificity of $95.8 \%$ (95\% CI 78.8-99.8\%) on the expense of a lower sensitivity of $85.7 \%(95 \%$ CI $42.1-99.6 \%)$ at a cutoff value of $212\left(\mathrm{mmoll}^{-1}\right)^{-1}$ (AUC is 0.964 , s.e.m. 0.03 and $95 \%$ CI $0.89-1.03$ ). The analysis of patients with PA due to an APA versus those with idiopathic hyperaldosteronism reveled that the $A R R \times$ SUSPPUP values were nonsignificantly higher in patients with APA compared with patients with idiopathic hyperaldosteronism $\left(1394.6 \pm 1556.9\left(\mathrm{mmoll}^{-1}\right)^{-1}\right.$ versus $881.9 \pm 668.4\left(\mathrm{mmoll}^{-1}\right)^{-1} ; \quad P=0.61$, nonparametric MannWhitney test).

In hypokalemic patients, ARR showed a sensitivity of $94.6 \%(95 \%$ CI $81.8-99.3 \%)$ and specificity of $90.0 \%(95 \%$ CI $55.5-99.8 \%)$ at a cutoff value of 35.8 (AUC is 0.947 , s.e.m. $0.04,95 \%$ CI $0.86-1.03$ ). In normokalemic patients, the ARR values displayed a lower sensitivity of $90.9 \%$ (95\% CI 70.8-98.9\%) combined with a lower specificity of $84.5 \%(95 \%$ CI $75.0-91.5 \%$ ) at a cutoff value of 24.6 (AUC is 0.956 , s.e.m. 0.02 , 95\% CI $0.92-0.99)$.

If the ARR (at a cutoff value of 26.35) was the only information used for detection of PA, four patients with PA (14.2\%) would have been missed and six patients (18.18\%) of EH would have been misdiagnosed as having PA. If the SUSPPUP ratio was used as a screening tool and a cutoff value of $5.95\left(\mathrm{mmoll}^{-1}\right)^{-1}$ considered, four patients with PA (14.2\%) would have been missed and five patients (15.15\%) with EH would have been classified as having PA. Applying the ARR $\times$ SUSPPUP formula with a cutoff value of 199.2 (mmoll $\left.{ }^{-1}\right)^{-1}$, out of the eight PA patients who had either a false-negative result for ARR or the SUSPPUP ratio, six were then correctly identified. None of the EH patients with false-positive results for SUSPPUP ratio had fulfilled the PA criteria, when $A R R \times S U S P P U P$ was applied. Only two $\mathrm{EH}$ patients (out of five) with ARR above 26.35 had higher values of ARR $\times$ SUSPPUP than the established cutoff point. Multiplying ARR with $A_{P L}$ or $A_{P L}{ }^{2}\left(A^{2} R R\right.$ and $A^{3} R R$ ) slightly increased the sensitivity at similar specificity values as compared with ARR.

At a cutoff value of $84.5 \mathrm{ngl}^{-1}$, serum aldosterone showed a good sensitivity (96.43\%), but low specificity $(69.7 \%)$, and the best negative predictive value (only one patient with PA had an $A_{P L}$ value below the cutoff point). However, when the cutoff value increases to $261 \mathrm{ngl}{ }^{-1}$, the specificity rises to $90.9 \%$, but the sensitivity declines to $53.57 \%$, with a smaller percentage of false-positive results in $\mathrm{EH}$ group and false-negative results in PA group.

The best specificity (100\%) and positive predictive value presented the $A_{U R} / R$ ratio at a cutoff value of $10.95 \mu \mathrm{g}$ per $24 \mathrm{~h}$ per $n g l^{-1}$, although with relatively low sensitivity. Multiplying the $A_{U R} / R$ ratio with $A_{U R}$ or with $A_{U R}{ }^{2}\left(A_{U R}{ }^{2} / R, A_{U R}{ }^{3} / R\right)$, gave the same sensitivity value and the specificity gradually decreased.

Adding $A_{U R}$ or $A_{U R} / R$ ratio as a second diagnostic criterion to the $A R R \times S U S P P U P$ formula, the specificity increases up to $100 \%$ (all the $\mathrm{EH}$ patients with false-positive results for the ARR $\times$ SUSPPUP ratio, were negative for the $A_{U R} / R$ ratio, at the above mentioned cutoff). 
Table 3 The investigated parameters, cutoff values chosen for the best sensitivity and specificity and positive and negative predictive values

\begin{tabular}{|c|c|c|c|c|c|c|c|c|}
\hline Test & Cutoff value & $A U C$ & s.e.m. & $95 \% \mathrm{Cl}$ & $\begin{array}{l}\text { Sensitivity in \% } \\
\quad(95 \% \mathrm{Cl})\end{array}$ & $\begin{array}{c}\text { Specificity in \% } \\
(95 \% \mathrm{Cl})\end{array}$ & $P P V$ & $N P V$ \\
\hline ARR $\times$ SUSPPUP $\left(\left(\mathrm{mmoll}^{-1}\right)^{-1}\right)$ & 199.2 & 0.977 & 0.01 & $0.94-1.00$ & $92.3(74.9-99.0)$ & $93.9(79.7-99.2)$ & 92.3 & 88.6 \\
\hline SUSPPUP $\left(\left(\mathrm{mmol} \mathrm{I}^{-1}\right)^{-1}\right)$ & 5.95 & 0.873 & 0.04 & $0.780-0.967$ & $84.6(65.1-95.6)$ & $82.3(65.5-93.2)$ & 81.5 & 87.1 \\
\hline ARR & 26.35 & 0.934 & 0.02 & $0.87-0.99$ & $85.7(67.3-96.0)$ & $81.8(64.5-93.0)$ & 80.0 & 87.1 \\
\hline$A_{U R}(\mu g$ per $24 \mathrm{~h})$ & 0.255 & 0.868 & 0.05 & $0.53-0.98$ & $94.7(73.9-99.8)$ & $62.5(35.4-84.8)$ & 73.9 & 83.3 \\
\hline$A_{U R} / R\left(\mu g\right.$ per $24 \mathrm{~h}$ per $\left.n g I^{-1} \times 10^{2}\right)$ & 10.95 & 0.919 & 0.05 & $0.82-1.01$ & $84.2(60.4-96.6)$ & $100(79.4-100)$ & 100.0 & 84.2 \\
\hline$A_{U R}{ }^{2} / R\left(\mu g^{2}\right.$ per $24 h$ per $\left.n g I^{-1}\right)$ & 2.41 & 0.927 & 0.04 & $0.84-1.01$ & $84.2(60.4-96.6)$ & 93.7 (69.7-99.8) & 94.1 & 83.3 \\
\hline$A_{U R}{ }^{3} / R\left(\mu g^{3}\right.$ per $24 h$ per $n g I^{-1}$ ) & 0.765 & 0.930 & 0.04 & $0.85-1.01$ & $84.2(60.4-96.6)$ & $87.5(61.6-98.4)$ & 88.8 & 82.3 \\
\hline$A_{U R} \times \mathrm{Na}\left(\mu \mathrm{g} \times \mathrm{mmol} \times 10^{-1}\right.$ per day $)$ & $\begin{array}{l}4.6 \\
3.09\end{array}$ & 0.807 & 0.07 & $0.66-0.95$ & $\begin{array}{l}78.9(54.4-93.9) \\
84.2(60.4-96.6)\end{array}$ & $\begin{array}{l}53.5(26.6-78.7) \\
50.0(24.6-75.3)\end{array}$ & $\begin{array}{l}68.2 \\
64.3\end{array}$ & $\begin{array}{l}66.7 \\
83.3\end{array}$ \\
\hline $\begin{array}{l}\text { THA } \times \mathrm{Na} / \mathrm{R}(\mu \mathrm{g} \times \mathrm{mmol} \text { per } \\
\left.24 \mathrm{~h} \text { per } \mathrm{ng}^{-1}\right)\end{array}$ & 1.55 & 0.876 & 0.04 & $0.786-0.967$ & $92.6(75.7-99.1)$ & $68.7(50.0-83.9)$ & 71.4 & 91.3 \\
\hline A18G/R ( $\mu$ g per $24 \mathrm{~h}$ per $n g I^{-1}$ ) & 2.1 & 0.902 & 0.05 & $0.79-1.00$ & $93.7(69.8-99.8)$ & $75.0(47.6-92.7)$ & 78.9 & 92.8 \\
\hline THA/R ( $\mu$ g per $24 \mathrm{~h}$ per $n g I^{-1}$ ) & 12.75 & 0.907 & 0.03 & $0.83-0.98$ & $89.3(71.8-97.7)$ & $75.0(56.6-88.5)$ & 75.7 & 88.5 \\
\hline A18G ( $\mu$ g per $24 h)$ & 12.5 & 0.834 & 0.07 & $0.66-0.97$ & $75.0(47.6-92.7)$ & $75.0(47.6-92.7)$ & 75.0 & 57.1 \\
\hline $\mathrm{A} 18 \mathrm{G} \times \mathrm{Na}\left(\mu \mathrm{g} \times \mathrm{mmol} \times 10^{-2}\right.$ per day $)$ & 8.75 & 0.769 & 0.08 & $0.60-0.93$ & $87.5(61.6-98.4)$ & $56.2(29.8-80.2)$ & 66.7 & 61.5 \\
\hline \multirow{2}{*}{$\begin{array}{l}\mathrm{A} 18 \mathrm{G} \times \mathrm{Na} / \mathrm{R}((\mu \mathrm{g} \times \mathrm{mmol} \\
\left.\left.24 \mathrm{~h} \text { per }\left.\mathrm{ng}\right|^{-1}\right) \times 10^{-2}\right)\end{array}$} & 2.0 & 0.841 & 0.06 & $0.707-0.975$ & $87.5(61.6-98.4)$ & $50.0(24.6-75.3)$ & 63.6 & 58.3 \\
\hline & 2.95 & & & & $81.2(54.4-95.9)$ & $68.7(41.3-89.0)$ & 72.2 & 78.6 \\
\hline$A_{P L}\left(n g I^{-1}\right)$ & 84.5 & 0.881 & 0.04 & $0.794-0.967$ & $96.4(81.7-99.9)$ & $69.7(51.3-84.4)$ & 73.0 & 95.8 \\
\hline $\mathrm{R}\left(\mathrm{ng} \mathrm{I}^{-1}\right)$ & 4.7 & 0.775 & 0.06 & $0.651-0.899$ & $82.1(63.1-93.9)$ & $72.7(54.4-86.7)$ & 71.9 & 82.7 \\
\hline
\end{tabular}

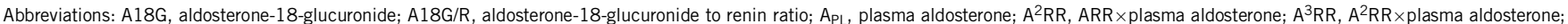
ARR aldosterone to renin ratio; $A_{U R}$, urinary unconjugated (free) aldosterone; $A_{U R} / R$, urinary unconjugated (free) aldosterone to renin ratio; $A U C$, area under curve; $C I$, confidence interval; $N a$, ARR aldosterone to renin ratio; $A_{U R}$, urinary unconjugated (free) aldosterone; $A_{U R} / R$, urinary unconjugated (free) aldosterone to renin ratio; $A U C$, area under curve; Cl, confidence interval; $\mathrm{Na}$,
sodium; NPV, negative predictive value; PPV, positive predictive value; R, renin; s.e.m., standard error of the mean; SUSPPUP, serum sodium: urinary sodium/(serum potassium) ${ }^{2}$ : urinary potassium
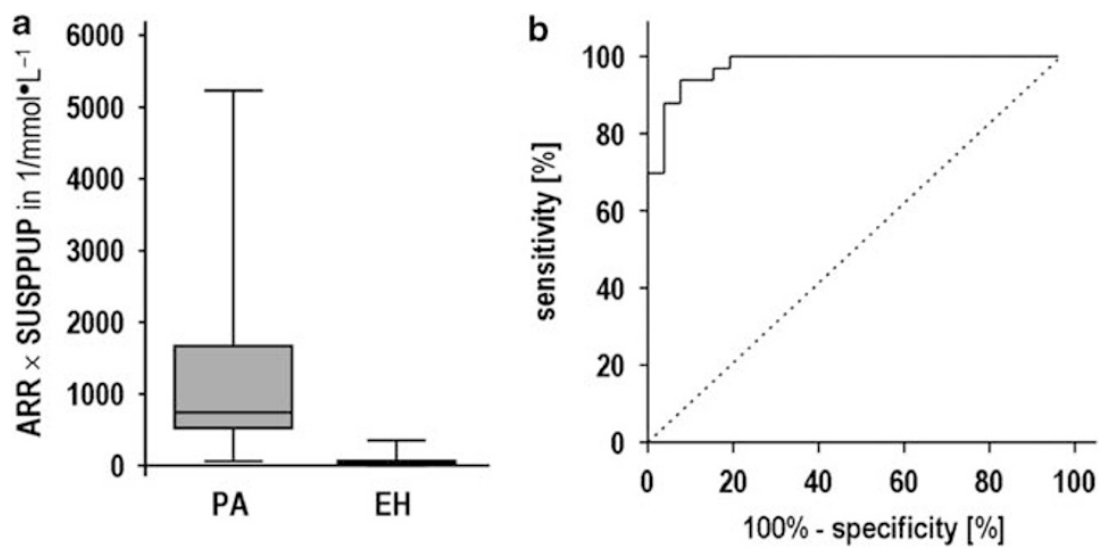

Figure 1 Panel a shows the distribution of the aldosterone-to-renin ratio (ARR) multiplied by the sodium to urinary sodium to (potassium) ${ }^{2}$ to urinary potassium (SUSPPUP) ratio in the group of patients with primary aldosteronism (PA) and essential hypertension (EH). Panel $\mathbf{b}$ gives a receiver-operating characteristic curve of the ARR multiplied by the SUSPPUP ratio in the distinction of individuals with PA from patients with EH.

Dividing urinary free aldosterone and its metabolites by the plasma renin concentration $\left(\mathrm{A}_{\mathrm{UR}} / \mathrm{R}, \mathrm{THA} / \mathrm{R}, \mathrm{A} 18 \mathrm{G} / \mathrm{R}\right)$ led to a better sensitivity. Correcting the urinary concentrations of free aldosterone and its metabolites for sodium diuresis did not improve sensitivity or specificity of the tests.
Though BBL/Clon tended to lower renin concentrations and elevated the ARR, these trends were not significant (Table 5). However, treatment with ACEI/ARB was associated with a significant higher renin concentration that lowered the ARR in EH patients (Table 5). This phenomenon was not visible in subjects with PA. Patients with 
Table 4 The best correlation that achieved the combined urinary secretion of THA and A18G

\begin{tabular}{lcccc}
\hline & $T H A+A 18 G$ & $T H A+A 18 G+A_{U R}$ & $A_{U R}$ & $T H A$ \\
\hline Correlation with ARR $\times$ SUSPPUP & 0.6315 & 0.6262 & 0.5446 & 0.5243 \\
$95 \% \mathrm{Cl}$ & $0.3780-0.7969$ & $0.3198-0.8140$ & $0.2376-0.7527$ & 0.5384 \\
$P$-value & $<0.0001$ & 0.0004 & 0.001 & $0.7183-0.7034$ \\
\hline
\end{tabular}

Abbreviations: A18G, aldosterone-18-glucuronide; $\mathrm{A}_{U R}$, urinary unconjugated (free) aldosterone; THA, tetrahydroaldosterone; $\mathrm{Cl}$, confidence interval.

Table 5 Antihypertensive medication had little influence on ARR and SUSPPUP ratio

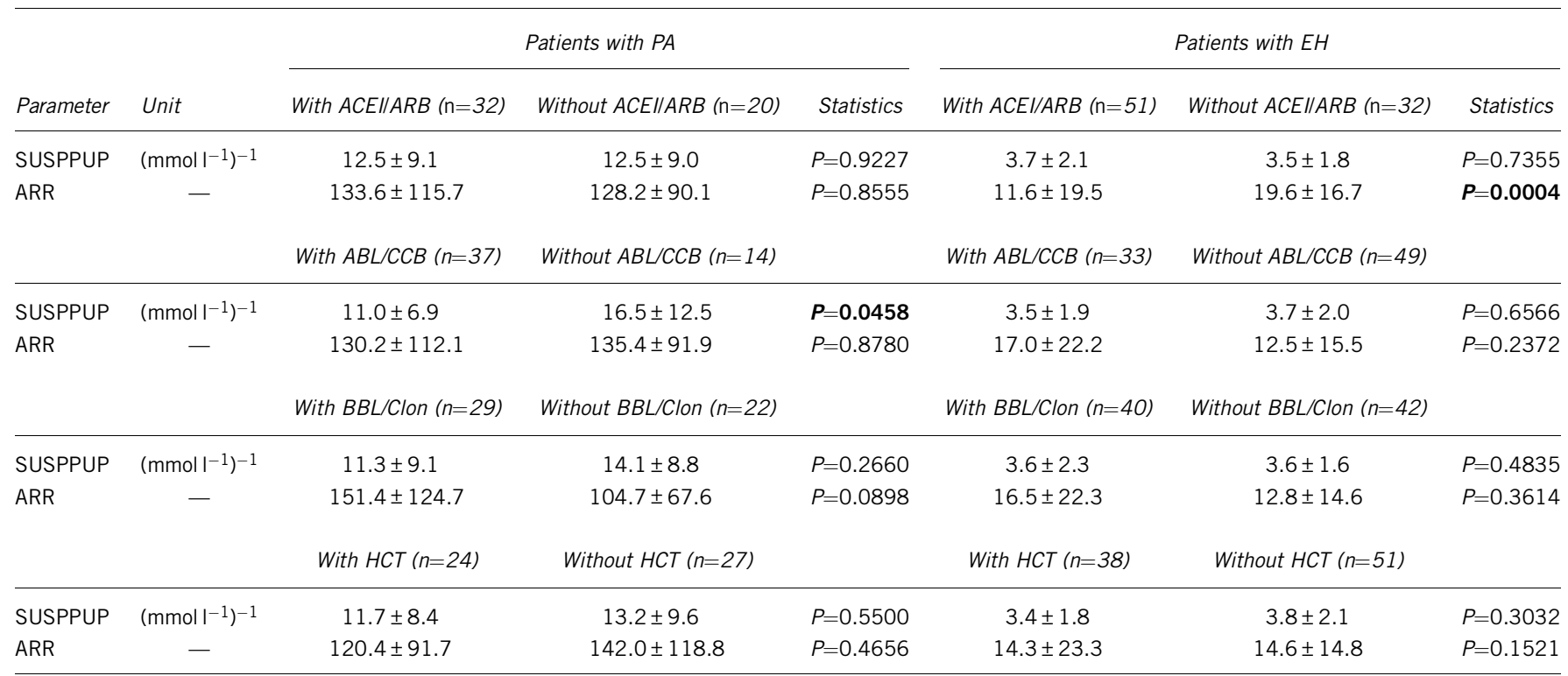

Abbreviations: ABL, alpha 1 -receptor blocker; $\mathrm{ACEI}$, angiotensin-converting enzyme inhibitor; ARB, angiotensin II type 1 receptor blocker; ARR, aldosterone to renin ratio; $\mathrm{BBL}$, beta-blocker; CCB, calcium channel blocker; Clon, antihypertensive drugs of the clonidine type; EH, essential hypertension; HCT, diuretics of the thiazide type; PA, primary aldosteronism; SUSPPUP, sodium to urinary sodium to (potassium) $)^{2}$ to urinary potassium ratio.

The differences were greater in patients with essential hypertension than in patients with primary aldosteronism, except for the treatment with $A B L / C C B$, where the difference between the patients on treatment was significantly lower than that between the patients without this medication.

PA, who were on calcium-channel blocker or $\alpha$-receptor blockers, had a lower SUSPPUP ratio, a trend that was already significant (Table 5). However, other parameters were not changed by treatment with calcium-channel blocker. Treatment with HCT-type diuretics did not interfere with the SUSPPUP ratio or ARR, both in patients with $\mathrm{PA}$ and $\mathrm{EH}$ (Table 5).

\section{DISCUSSION}

Primary aldosteronism is present in up to $13 \%$ of hypertensive patients and is associated with additional cardiovascular morbidity. ${ }^{24,25}$ The early detection of PA in the hypertensive population is beneficial, because specific treatment approaches improve the associated mortality and morbidity. ${ }^{1,24,26}$

If the $A_{P L} / P R A$ ratio is used as a screening tool, the prevalence of PA in hypertensive patients varies between 9 and 13\%, depending on the patient population. $2,3,6,13,27,28$ However, there is still a lack of consensus regarding the appropriate method for determination of renin. PRA is dependent on the angiotensinogen concentration, as well as on renin concentration. Some studies in healthy volunteers reported a close correlation between PRA values and plasma renin concentrations, as determined by immunoassay. ${ }^{29}$ However, this correlation was not as close for low PRA and plasma renin concentrations, and in patients with different angiotensinogen concentrations. ${ }^{29}$

The pivotal role of aldosterone in the pathophysiology of $\mathrm{EH}$ is now well documented. ${ }^{30-32}$ Although $15 \%$ of patients with hypertension exhibit alterations in aldosterone regulation, they do not meet the criteria for PA. Suppression of renin is thought to be the earliest feature of PA. ${ }^{33}$ The use of $A_{P L} / P R A$ ratio in populations with high prevalence of low-renin hypertension may lead to a high number of false-positive results. ${ }^{33}$

Many studies investigated the effects of different antihypertensive drugs on the renin-angiotensin-aldosterone system and implicit on $A_{P L} /$ PRA ratio. Several studies concluded that mean $A_{P L}$ and PRA values are not significantly influenced by medication in patients with PA. ${ }^{17}$ Other data suggest that $A_{\mathrm{PL}} / \mathrm{PRA}$ ratio or ARR (using plasma renin concentration) are valid screening tools for PA in outpatients with ongoing antihypertensive treatment. ${ }^{11,13,34,35}$ The results of this study match well with the data of previously published studies showing that treatment with ACEI or ARB was associated with higher renin concentrations and a lower ARR.9,17,36 Of note, the effects were more profound in patients with EH. Accordingly, the SUSPPUP ratio was less influenced by treatment with ACEI or ARB in PA. This is probably because of the fact that the modulation of the reninangiotensin-aldosterone system by ACEI or ARB is not so relevant in PA, because here the renin-angiotensin-aldosterone system is already suppressed by the strong negative feedback. In addition, medication with BBL or Clon was associated with lower renin concentrations, elevating the ARR almost significantly. Although the difference between the treatment groups was higher in the $\mathrm{EH}$ population, the mathematical impact was greater in the PA group, probably for two reasons. First, renin is already decreased in PA and closer to zero than in $\mathrm{EH}$ and, second, aldosterone follows renin more 
closely in $\mathrm{EH}$ than in PA. Consequently, BBL/Clon treatment did not significantly interfere with the SUSPPUP ratio, because aldosterone secretion occurs to some extent independent of renin in PA. ${ }^{14}$ Patients under HCT therapy had higher renin levels than those who were not and aldosterone followed renin levels in $\mathrm{EH}$ patients but not in patients with PA, resulting in a lower ARR in PA patients, while the ARR remained stable in EH patients. Interestingly, the SUSPPUP ratios were not different between the treatment groups, thus indicating that HCT does not favor kaliuresis over natriuresis. An unexpected finding was that the SUSPPUP ratio was lower in PA patients who were on calcium-channel blocker or $\alpha$-receptor blocker treatment, although a significant effect on the ARR was not seen. This fact deserves further attention.

In our study, $A^{3} R R$ and $A^{2} R R$ showed a sensitivity and specificity that is similar to the data reported by Seiler et al. ${ }^{17}$, despite different laboratory assays and cutoff levels used. Previous studies showed the good accuracy for these tests using PRA values. Many patients with PA have a suppressed PRA, with values below $1 \mathrm{ng} \mathrm{ml}^{-1} \mathrm{~h}^{-1}$, therefore enhancing the $\mathrm{A}_{\mathrm{PL}} / \mathrm{PRA}$ ratio. ${ }^{14,17}$

The good accuracy of ARR is confirmed by other studies, in which plasma renin concentration was used with $1 \mathrm{ngl}^{-1}$ as lower detection limit of the laboratory assay. ${ }^{37}$

The same principle was applied for $A_{U R} / R$ ratio. Multiplying the ratio with $A_{U R}$ or $A_{U R}{ }^{2}$, the AUC increased, but the specificity decreased gradually at constant sensitivity. In conclusion, in contrast with $A^{2} R R$ and $A^{3} R R$, the $A_{U R}{ }^{2} / R$ and $A_{U R}{ }^{3} / R$ ratios do not seem to improve the detection of PA patients.

Urinary sodium concentration depends on many factors such as dietary sodium intake or drugs such as diuretics, ACEIs and many more. Therefore, our results should be interpreted in a clinical context. The SUSPPUP ratio is a mathematical expression of sodium retention related to renal potassium loss. Potassium squared was introduced in the formula because, in addition to the renal loss in exchange for sodium, in PA, potassium is diluted because of the hypervolemic state, thus becoming a specific parameter. ${ }^{21}$ As SUSPPUP ratio was introduced as an easy tool, useful also in the screening for PA, the present study evaluated the value of this test in patients with ongoing antihypertensive treatment. The results of our study showed a good sensitivity and specificity that was, however, slightly lower than those previously reported. ${ }^{21}$

Applying the ARR $\times$ SUSPPUP formula, sensitivity and specificity further increased as compared with ARR and SUSPPUP ratio alone. The formula reflects the inadequate secretion of aldosterone, along with the estimation of mineralocorticoid action. Interestingly, the optimal cutoff value did not differ between hypokalemic and normokalemic patients. So far, this test seems to be the most reliable in the case identification of PA patients, with the smallest percentage of falsepositive and -negative results. Therefore, the ARR $\times$ SUSPPUP test is worth further prospective evaluation. By the way, the ARR lost specificity and sensitivity when applied to hypokalemic patients. One explanation could be the small proportion of hypokalemic patients with $\mathrm{EH}$.

Most laboratories measure excretion of A18G, as 'urinary aldosterone'. The discrepancies regarding the reported values of $A_{U R}$ are because of the fact that in the present study, free urinary aldosterone is determined separately from A18G.

The urinary aldosterone metabolites and free urinary aldosterone determined in 24-h urine sample reflect the endogenous secretion of aldosterone better than serum aldosterone concentration in one random blood sample. The present study showed that $A_{U R} / R$ has a higher specificity and sensitivity as compared with $\mathrm{A}_{\mathrm{PL}}$.
Our results showed that urinary aldosterone metabolites had a rather limited performance with respect to sensitivity or specificity and are not suitable as screening tests for PA. This is in contrast to the reports by other groups. ${ }^{18}$ In addition, sensitivity and specificity could not be improved by the correction for sodium excretion. However, the correction of urinary aldosterone metabolites, especially free aldosterone, for plasma renin concentration rendered acceptable profiles and a very high specificity. This is in line with previous observations that also showed that the $A_{U R} / R$ ratio is also of good predictive value whether or not hypertension is reversible after adrenalectomy for APA. ${ }^{19,20}$

As a limitation, most of the PA patients were under antihypertensive therapy at the time of screening and the present retrospective study did not assess the influence of various antihypertensive drugs on the laboratory parameters. In addition, sodium intake also influences the ARR and the current study did not analyze the effect of low sodium intake versus high sodium intake on the aldosterone excess parameters in the same patients. However, we have liberalized the sodium intake during the diagnostic procedure and as noted above, correcting urinary aldosterone (or metabolite) secretion for sodium diuresis did not improve sensitivity or specificity. Therefore, such a correction for sodium may not be useful in the characterization of aldosterone excess, given a certain amount of daily sodium consumption.

A second important point is that almost a half of the patients with PA in this study were diagnosed with idiopathic hyperaldosteronism. In these patients, there was no histological confirmation of an adrenal adenoma with cells of the zona glomerulosa type. In addition, EH patients were ruled out to have PA when they suppressed their aldosterone in the confirmatory test below $50 \mathrm{ngl}^{-1}$. Therefore, we cannot be sure that some of these cases may develop or 'cross the cutoff values' for PA in the future.

In conclusion, the data provided by the present study favors $A R R \times$ SUSPPUP as the most sensitive and specific screening tool for $P A$, followed by $A^{3} R R$ and $A_{U R} / R$. The combination of $A R R \times S U S P P U P$ with the $A_{U R} / R$ ratio increased the diagnostic specificity for PA to $100 \%$ in our cohort.

\section{ACKNOWLEDGEMENTS}

This work was supported by grants from the Institute for International Communication, Heinrich-Heine-University Duesseldorf and by the Georg Strohmeyer-Stiftung, Duesseldorf, Germany, to MB and HSW.

1 Funder JW, Carey RM, Fardella C, Gomez-Sanchez CE, Mantero F, Stowasser M, Young Jr WF, Montori VM. Case detection, diagnosis and treatment of patients with primary aldosteronism: an Endocrine Society Clinical Practice Guideline. J Clin Endocrinol Metab 2008; 93: 3266-3281.

2 Mulatero P, Stowasser M, Loh KC, Fardella CE, Gordon RD, Mosso L, Gomez-Sanchez $\mathrm{CE}$, Veglio F, Young Jr WF. Increased diagnosis of primary aldosteronism, including surgically correctable forms, in centers from five continents. J Clin Endocrinol Metab 2004; 89: 1045-1050.

3 Gordon RD, Stowasser M, Tunny TJ, Klemm SA, Rutherford JC. High incidence of primary aldosteronism in 199 patients referred with hypertension. Clin Exp Pharmacol Physiol 1994; 21: 315-318.

4 Ribeiro MJS, Figueiredo Neto JA, Memoria EV, Lopes Mde C, Faria Mdos S, Salgado Filho N, Oliveira TC. Prevalence of primary hyperaldosteronism in a systemic arterial hypertension league. Arq Bras Cardiol 2009; 92: 37-43.

5 Nishikawa T, Saito J, Omura M. Prevalence of primary aldosteronism: should we screen for primary aldosteronism before treating hypertensive patients with medication? Endocrine J 2007; 54: 487-495.

6 Rossi GP, Bernini G, Caliumi C Desideri G, Fabris B, Ferri C, Ganzaroli C, Giacchetti G, Letizia C, Maccario M, Mallamaci F, Mannelli M, Mattarello MJ, Moretti A, Palumbo G, Parenti G, Porteri E, Semplicini A, Rizzoni D, Rossi E, Boscaro M, Pessina AC, Mantero F, PAPY Study Investigators. A prospective study of the prevalence of primary aldosteronism in 1125 hypertensive patients. J Am Coll Cardiol 2006; 48: 2293-2300. 
7 Bernini G, Moretti A, Argenio G, Salvetti A. Primary aldosteronism in normokalemic patients with adrenal incidentalomas. Eur J Endocrinol 2002; 146: 523-529.

8 Young WF. Primary aldosteronism: renaissance of a syndrome. Clin Endocrinol 2007; 66: 607-618.

9 Seifarth C, Trenkel S, Schobel H, Hahn EG, Hensen J. Influence of antihypertensive medication on aldosterone and renin concentration in the differential diagnosis of essential hypertension and primary aldosteronism. Clin Endocrinol 2002; 57. 457-465.

10 Nishizaka MK, Pratt-Ubunama M, Zaman MA, Cofield S, Calhoun DA. Validity of plasma aldosterone-to-renin activity ratio in African American and white subjects with resistant hypertension. Am J Hypertens 2005; 18: 805-812.

11 Schwartz GL, Turner ST. Screening for primary aldosteronism in essential hypertension: diagnostic accuracy of the ratio of plasma aldosterone concentration to plasma renin activity. Clin Chem 2005; 51: 386-394.

12 Doi SAR, Abalkhail S, AI-Qudhaiby MM, Al-Humood K, Hafez MF, AI-Shoumer KAS. Optimal use and interpretation of the aldosterone renin ratio to detect aldosterone excess in hypertension. J Hum Hypertens 2006; 20: 482-489.

13 Gallay BJ, Ahmad S, Xu L, Toivola B, Davidson RC. Screening for primary aldosteronism without discontinuing hypertensive medications: plasma aldosterone-renin ratio. $\mathrm{Am} \mathrm{J}$ Kidney Dis 2001; 37: 699-705.

14 Willenberg HS, Schinner S, Ansurudeen I. New mechanisms to control aldosterone synthesis. Horm Metab Res 2008; 40: 435-441.

15 Weinberger MH, Fineberg NS. The diagnosis of primary aldosteronism and separation of two major subtypes. Arch Intern Med 1993; 153: 2125-2129.

16 Young WF. Primary aldosteronism. Management issues. Ann NY Acad Sci 2002; 970 : 61-76.

17 Seiler L, Rump L, Reincke M, Pavenstädt H, Borm K, Slawik M, Schulte-Mönting J, Beuschlein F. Diagnosis of primary aldosteronism: value of different screening parameters and influence of antihypertensive medication. Eur J Endocrinol 2004; 150: 329-337.

18 Abdelhamid S, Blomer R, Hommel G, Haack D, Lewicka S, Fiegel P, Krumme B. Urinary tetrahydroaldosterone as a screening method for primary aldosteronism: a comparative study. Am J Hypertens 2003; 16: 522-530.

19 Mourad JJ, Girerd X, Milliez P, Lopez-Sublet M, Lejeune S, Safar ME. Urinary aldosterone-to- active-renin ratio: a useful tool for predicting resolution of hypertension after adrenalectomy in patients with aldosterone-producing adenomas. Am J Hypertens 2008; 21: 742-747.

20 Bernini G, Moretti A, Orlandini C, Berti P, Miccoli P, Bardini M, Taurino C, Bernini M, Salvetti A. Plasma and urinary aldosterone to plasma renin activity ratio in the diagnosis of primary aldosteronism. J Hypertens 2008; 26: 981-988.

21 Willenberg HS, Kolentini C, Quinkler M, Cupisti K, Krausch M, Schott M, Scherbaum WA. The serum sodium to urinary sodium to (serum potassium) ${ }^{2}$ to urinary potassium (SUSPPUP) ratio in patients with primary aldosteronism. Eur J Clin Invest 2009; 39: 43-50.

22 Vecsei P, Abdelhamid S, Mittelstaedt GV, Lichtwald K, Haack D, Lewicka S. Aldosterone metabolites and possible aldosterone precursors in hypertension. J Steroid Biochem 1983; 19: 345-351.
23 Maser-Gluth C, Reincke M, Allolio B, Schulze E. Metabolism of glucocorticoids and mineralocorticoids in patients with adrenal incidentalomas. Eur J Clin Invest 2000; 30: 83-86.

24 Rossi G, Boscaro M, Ronconi V, Funder JW. Aldosterone as a cardiovascular risk factor. Trends Endocrinol Metab 2005; 16: 104-107.

25 Born-Frontsberg E, Reincke M, Beuschlein F, Quinkler M, Participants of German Conn's Registry. Tumor size of Conn's adenoma and comorbidities. Horm Metab Res 2009; 41: 785-788.

26 Schirpenbach C, Seiler L, Maser-Gluth C, Rüdiger F, Nickel C, Beuschlein F, Reincke M. Confirmatory testing in normokalaemic primary aldosteronism: the value of the saline infusion test and urinary aldosterone metabolites. Eur J Endocrinol 2006; 154: 865-873.

27 Fardella CE, Mosso L, Gomez-Sanchez C, Cortes P, Soto J, Gomez L, Pinto M, Huete A, Oestreicher E, Foradori A, Montero J. Primary hyperaldosteronism in essential hypertensives: prevalence, biochemical profile and molecular biology. J Clin Endocrinol Metab 2000; 85: 1863-1867.

28 Jansen PM, Boomsma F, van den Meiracker AH, Dutch ARRAT investigators. Aldosterone-to-renin ratio as a screening test for primary aldosteronism-The Dutch ARRAT Study. Neth J Med 2008; 66: 220-228.

29 Campbell DJ, Nussberger J, Stowasser M, Jan Danser AH, Morganti A, Frandsen E, Menard J. Activity assays and immunoassays for plasma renin and prorenin: information provided and precautions necessary for accurate measurement. Clin Chem 2009; 55: 867-877.

30 Grim CE. Evolution of diagnostic criteria for primary aldosteronism: why is it more common in 'drug-resistant' hypertension today? Curr Hypertens Rep 2004; 6: 485-492.

31 Ho JT, Keogh JB, Bornstein SR, Ehrhart-Bornstein M, Lewis JG, Clifton PM, Torpy DJ. Moderate weight loss reduces renin and aldosterone but does not influence basal or stimulated pituitary-adrenal axis function. Horm Metab Res 2007; 39: 694-699.

32 Vinciguerra M. Aldosterone, but not increased $\mathrm{Na}+$ influx or NF-kappaB activation, increases kidney-specific WNK1 gene expression in renal collecting duct cells. Horm Metab Res 2009; 41: 67-70.

33 Rayner B. Primary aldosteronism and aldosterone-associated hypertension. J Clin Pathol 2008; 61: 825-831.

34 Trenkel S, Seifarth C, Schobel H, Hahn EG, Hensen J. Ratio of serum aldosterone to plasma renin concentration in essential hypertension and primary aldosteronism. Exp Clin Endocrinol Diabetes 2002; 110: 80-85.

35 Niizuma S, Nakahama H, Kamide K, Fukuchi K, Iwanaga Y, Nakata H, Yoshihara F, Horio T, Nakamura S, Kawano Y. The cutoff value of aldosterone-to-renin ratio for the diagnosis of primary aldosteronism in patients taking antihypertensive medicine. Clin Exp Hypertens 2008; 30: 640-647.

36 Mulatero P, Rabbia F, Milan A, Paglieri C, Morello F, Chiandussi L, Veglio F. Drug effects on aldosterone/plasma renin activity ratio in primary aldosteronism. Hypertension 2002; 40: 897-902.

37 Unger N, Lopez Schmidt I, Pitt C, Walz MK, Philipp T, Mann K, Petersenn S. Comparison of active renin concentration and plasma renin activity for the diagnosis of primary hyperaldosteronism in patients with an adrenal mass. Eur $J$ Endocrinol 2004; 150: 517-523. 